\title{
Aportes conceptuales sobre las prácticas sociales en la feria callejera. El tema de la confianza social ${ }^{*}$
}

\section{Eduardo Chávez Molina**}

Una sociedad caracterizada por la reciprocidad generalizada es más eficiente que otra desconfiada, por la misma razón que la eficiencia del dinero es mayor que la del trueque. Si no tenemos que compensar cada intercambio al instante, podremos realizar muchas más cosas. La fiabilidad es lubricante de la vida social (Putnam, 2002).

Resumen: La expansión de las actividades informales de feriantes en el conurbano bonaerense constituye una de las manifestaciones del fenómeno de la heterogeneidad no regulada del aprovisionamiento de bienes de consumo, que a su vez, emergen como intermediarios económicos que atienden a necesidades y demandas de consumidores territorialmente segregados y no articulados a los mercados formales, en su mayoría.

Es en ese sentido que el presente artículo quiere rescatar y señalar los componentes centrales que asoman en un proceso que parte con el supuesto de que la confianza constituye un elemento central cuando otras formas que actuarían como garante de las interacciones aparecen débiles, ausentes o no primordiales como garantía de las interacciones sociales, como es el caso de la feria de Solano.

Palabras clave: prácticas sociales, mercado informal, confianza social, marginalidad

\section{Conceptual contributions on social practices $\mathbf{i}$ n the street fair.The subject of social confidence}

Abstract: The expansion of peddlers' informal activities in the Great Buenos Aires region constitutes one of the expressions of the phenomenon of heterogeneity not regulated the provision of consumer goods. Peddlers emerge as

\footnotetext{
* Este estudio se inscribe en la investigación "Prácticas sociales en el trabajo informal” del equipo Cambio estructural y Desigualdad social, del Instituto de Investigaciones Gino Germani, que dirige el Doctor Agustín Salvia. El diseño de la investigación implicó el desarrollo de técnicas cualitativas (grupos de discusión, entrevistas en profundidad, y observación participante) y técnicas cuantitativas (encuestas a feriantes, a consumidores, y conteo de puestos). En el presente trabajo se presentan principalmente lo resultados teóricos de la investigación.

*Universidad de Buenos Aires, Buenos Aires, Argentina. Email: echavez@mail.retina.ar
} 
economic intermediaries who cater to the needs and demands of a population of consumers territorially segregated and largely disconnected from formal markets.

It is in this sense that the present article aims to recover and indicate the central components that appear in this process of intermediation. A process that starts with the assumption that trust constitutes a central element when other forms that could act as guarantee of social interactions turn out to be weak, absent or not primary, as it is the case in Solano's fair. marginality

Key words: Social practices, informal market, social confidence,

\section{Contribuições conceituais sobre as práticas sociais na feira de rua. $\mathbf{O}$ tema da confiança social}

Resumo: A expansão das atividades informais de feriantes no conurbano bonaerense constitui uma das manifestações do fenômeno da heterogeneidad não regulada do abastecimento de bens de consumo, que a sua vez, emergem como intermediários econômicos que atendem a necessidades e demandas de consumidores territorialmentesegregados e não articulados aos mercados formais, em sua maioria. É nesse sentido que o presente artigo quer resgatar e assinalar os componentes centrais que assomam num processo que parte com o suposto de que a confiança constitui um elemento central quando outras formas que actuariam como garantia das interações aparecem débis, ausentes ou não primordiais, como é o caso da feira de Solano. marginalidade

Palavras-chave: práticas sociais, mercado informal, confiança social,

Recibido: 18.11.2008

Aceptado: 10.06.2009

$* * *$

\section{Introducción}

Parafraseando a Marx: "el mundo se presenta como un cúmulo de mercancías” (Marx, 1994), el esfuerzo teórico y empírico de las ciencias sociales en general y desde la sociología en particular implica la descripción, y explicación del mundo social, el cual se enfrenta con la necesidad de superar el primer obstáculo de comprensión a partir fenómenos que aparecen en la superficie de la comprensión. Ese es el lugar que le cabe a las ferias callejeras, y a sus prácticas cotidianas. Ante nuestros ojos este espacio socio-comercial aparece como una multiplicidad de intercambios de mercaderías que proliferan en una maraña de puestos, en los cuales hombres y mujeres, dan forma social al espacio urbano donde se desarrolla la feria.

Los días miércoles y sábados de cada semana, en San Francisco Solano, en la zona sur del conurbano bonaerense, se desarrolla una de las ferias urbanas más grande, en cantidad y extensión, de la provincia de Buenos Aires. Asisten a la misma, vecinos y comerciantes a comprar e intercambiar productos, a lo largo de 25 cuadras. Un escenario social donde se 
configura una red de articulaciones, con mayor o menor grado de formalidad, y en la cual los vínculos basados en la confianza aparecerían como los sostenedores de las prácticas económicas en su interior, reemplazando o sobreponiéndose a las regulaciones públicas, garantizando los intercambios y arbitrando la continuidad y perdurabilidad de los mismos en el espacio callejero.

Las ferias callejeras no solo representan aquellos espacios físicos de intermediación comercial de productos y servicios, entre productores, intermediarios y consumidores, sino que también se constituyen en espacios sociales donde se condensan condiciones socio-estructurales, institucionales y/o subjetivas de segregación con o sin vinculación con los espacios formales, modernos y dinámicos. A la vez de conformar acuerdos tácitos o explícitos de “convivencia societal”, que permite regular el espacio y el tiempo social de la reproducción económica de la feria.

Es en ese sentido que el presente artículo quiere rescatar y señalar los componentes centrales que asoman en un proceso que parte con el supuesto de que la confianza constituye un elemento central cuando otras formas que actuarían como garante de las interacciones aparecen débiles, ausentes o no primordiales, como es el caso de la feria de Solano.

En los feriantes, el momento de llegada, que implica en muchos casos generar o imponer relaciones para asegurarse un lugar en la feria, garantizar la venta, regularizar las transacciones, entre otros fenómenos, permite apreciar los múltiples intercambios sociales que se ponen en juego para ser utilizados en el espacio público, y es uno de los motivos centrales de cierto conflicto y cierto proceso de cooperación social en la misma. Gestionar, armar o insertarse en una red de relaciones que les otorguen en el corto y mediano plazo beneficios implica conexiones útiles, contactos personales que aseguren o faciliten el acceso a este tipo de ocupaciones, y a un mejor posicionamiento físico al interior de la feria.

Desde allí es oportuno hablar de las prácticas sociales, dicha noción, se inscribe en el debate conceptual sobre una teoría de las prácticas, de la acción y los sentidos y orientaciones de la acción (Weber, 1993, Giddens 1986, Bourdieu 2004, Val Burris 1993, Alexander 1997 entre otros) y donde numerosos estudios han dado un rico y vasto aporte a las ciencias sociales, desde perspectivas teóricas y empíricas sobre la teoría de la acción.

\section{El capital social: eje posible de las estrategias de reproducción}

Desde vasta literatura sobre el tema de capital social (Espinosa, 1998; CEPAL, 1997; Beccaria, Katzman, Kessler, Golbert, Filgueira, 1999, Lin, 2001, Putnam 2002, Coleman 1990) se plantea que generalmente las raíces de la pobreza está en las desigualdades generadas por el mercado, (lugar de las relaciones de explotación) pero los sectores pobres tienen un potencial 
de atenuar estas desigualdades por medio de iniciativas personales, utilizando sus relaciones sociales para compensar su carencia de capital material. Desde esa perspectiva general, algunos autores enfatizan el potencial que tienen los sectores pobres para escapar de esa situación por medio de la utilización estratégica de sus activos en combinación con el apoyo de las organizaciones de la sociedad civil y el Estado (Beccaria, Katzman, Kessler, Golbert, Filgueira, 1999). De ahí se propugna la idea de estudiar las redes sociales, para entender el funcionamiento de colectivos sociales, organizaciones y comunidades.

En nuestro caso de los feriantes, se observa la construcción y recreación de sus prácticas cotidianas, validándose habitual y regularmente, lo cual implica poner en ejercicio, en la acción, y particularmente en los procesos de interacción social, un conjunto de habilidades, capacidades y relaciones que podemos englobarlo bajo el concepto de capital social. Bajo ese prisma, se encuentra una vasta literatura al respecto, y diferentes matices en torno al concepto que pasamos a continuación a enunciar.

\section{El intercambio social y la consolidación de la feria}

Un aspecto importante en el entramado social de la feria, es que los beneficios obtenidos, ya sea a través de las ventas, o las relaciones sociales que se gestan allí, pueden estar precedidas por la idea de la obtención de recompensas, como desde otra perspectiva plantea Bourdieu en el proceso de acumulación de capital (Bourdieu, P., Chamboredon J. C., Passeron J.C. 2004).

Bajo la idea de la recompensa, los actores actúan. Desde esa premisa, caben dos interrogantes centrales, qué beneficios acarrea y cómo los individuos acceden a él. Para Lin (Lin, 2001) en el marco de estas preguntas, y ligadas a los supuestos anteriores, se establecen algunas premisas: la principal es que los individuos invierten en relaciones sociales con la expectativa de obtener retornos y, con este fin, interactúan. El capital social es concebido como un activo social generado por las conexiones entre actores y constituido por recursos de los que se carece. En la medida en que los recursos que constituyen ese activo están insertos en las redes y sólo se capitalizan individualmente, el capital social no puede ser considerado como un bien público o colectivo. Es social porque deriva de interacciones (Lin, 2001). Las redes delimitan la cantidad y distribución de recursos que pueden ser accesibles y dan un marco para la ubicación y estabilidad de lazos de cada individuo. Es por esto que que "el capital social debe ser concebido como recursos accesibles a través de lazos sociales que ocupan lugares estratégicos y/o posiciones organizativas significativas. Operacionalmente, el capital social puede ser definido como "los recursos arraigados en redes sociales a los que unos actores acceden y los usan para acciones”. (Lin, 2001).

Siguiendo los aportes de Lin, plantea que los recursos del capital social pueden ser transferidos, movilizados: la red se entiende como la es- 
tructura que organiza recursos y posiciones estratégicas de individuos, la interacción, como medio de acceso a esos recursos y al acercamiento de posiciones; y la acción -o sus tipos- como orientación de la conducta para obtener retornos.

La acción se orienta instrumentalmente. Se entiende así que la interacción representa "no sólo la reunión de dos actores, sino la reunión de dos posiciones sociales que los actores ocupan; interactuar con un actor que posee más recursos, implica interactuar con una posición social con más recursos” (Lin, 2001).

En el mundo de la feria, la obtención de recompensas está presente en el proceso de intercambio, pero que las mismas no pueden ser pensadas sólo desde una lógica del beneficio económico, sino que en el beneficio común, que puede o no transformase en resultados positivos en futuro, en su carácter individual. Asimismo el ocupar un lugar disímil en la estructura de la feria, condiciona el posicionamiento en la red de la cual se es parte, y otorga sentido a intercambios equiparables entre los actores, pero que no significan lo mismo para cada actor.

Si la presencia de la administración pública implica un carácter híbrido a los esquemas regulatorios que pueden observarse en otros mercados, el valor que asume la pertenencia a una red, y la consolidación de la misma, se torna prioritario y casi excluyente, en el momento de analizar resultados y beneficios al interior de la feria.

\section{Las redes sociales y sus características}

Este tipo de análisis sobre las prácticas sociales, permite observar la mediación de las redes sociales en el posicionamiento en la estructura de la feria y el lugar que suple cuando encontramos débiles regulaciones en los intercambios o por otro lado compensando y legitimando las interacciones de carácter informal.

Un aporte interesante sobre el análisis de redes es el estudio de Granovetter (1985), sobre dos maneras básicas de analizar estas redes que giran en torno al individuo por medio de:

- su grado de conexión (la forma en los miembros de la red están conectados),

- sus vínculos, ya sean estos simples o múltiples.

Sobre este último punto vale la pena detenerse, en el sentido de analizar estas diferencias. Las redes con base en vínculos múltiples son aquellas en las que las personas o unidad familiar se relacionan con otros a través de compartir varias dimensiones de actividad (trabajo, religión, origen común, recreación). En contraste, una red con base en vínculos simples, el individuo o la familia se relaciona con otros en una dimensión de 
actividad, de manera tal, que por ejemplo, un individuo conoce a gente diferente en el trabajo que en el vecindario o en la iglesia.

En ese sentido, las comunidades que tienen como base a las personas y las familias con redes en vínculos simples serán menos integradas, pero también más pasibles de ser abiertas hacia fuera "ya que las redes aisladas contendrán relaciones variadas y dispersas” (Granovette, 1985:33). Este tipo de relación es lo que Granovetter (Ibid, 1985) llamó la solidez de los vínculos débiles. En su investigación sobre búsqueda de empleo, Granovetter señaló una de las ventajas más importantes de las redes con base en vínculos simples, que “se extienden más allá de la comunidad inmediata de compañeros de trabajo o de vecinos, donde la información sobre empleos de duplica rápidamente en personas y lugares que suministran nueva información y sirven para encontrar empleo (Granovetter, 1985). Al contrario, los vínculos sólidos restringen la búsqueda de empleo, pero lo hacen por medio de intermediarios y de la contratación por recomendación verbal que permite a los pobres y los no calificados conseguir trabajo al asentarse masivamente en empleos en pequeñas empresas que buscan mano de obra barata, pero fiable.

En los últimos años se ha considerado de vital importancia en la gestión de las pequeñas empresas el tema de las redes, bajo un abordaje transdisciplinario, que recorre principalmente la economía y la sociología, y que permite entender, desde esta lógica, los factores que habilitan o no perfomances positivos o negativos en este tipo de unidades económicas. (Szarka, 1998).

Esta es una de las interpretaciones que se propone para caracterizar al sector “informal”, aunque el concepto de redes tiene su origen en las relaciones organizacionales de negocios dentro de la literatura económica. Si tenemos en cuenta la situación particular de funcionamiento de las actividades informales, tal como se describirá más adelante, la formación de redes resulta vital para su supervivencia, y también para los resultados económicos que logre.

Una red debe ser entendida "generalmente como un tipo específico de relación, que vincula a un conjunto de personas, objetos o eventos” (Szarka, 1998:320). Dichas relaciones pueden estar basadas en personas o instituciones, y el punto de referencia puede variar entre la unidad económica y el agente como tal. Son clasificadas de acuerdo a la proximidad de esas redes, al carácter institucional y al tipo de intercambio.

Las relaciones sociales o de proximidad se centran principalmente en las características del actor económico, en sus lazos de amistad basados en la confianza. Se expresan en la familia, los amigos, los vecinos, que permiten un primer nivel de relaciones, las cuales pueden articularse en forma directa o indirecta con la unidad económica. En muchos casos, la familia constituye el núcleo del cual se obtiene la fuerza de trabajo necesaria para el funcionamiento de la actividad económica, y además el lugar donde se toman las decisiones. 
Las relaciones institucional burocráticas o de comunicación, están constituidas por el conjunto de aquellas organizaciones con que la unidad económica establece vínculos no comerciales que dan forma a sus actividades de negocio, como consultores y asesores, gobiernos locales y centrales, y sus agentes. Las relaciones pueden estar ceñidas a regulaciones de los diferentes niveles de gobierno; pueden ser de asistencia y promoción, pública o privada, y de información, que permite tomar en mejores condiciones estrategias a seguir por parte de la unidad económica.

Y por último las relaciones de intercambio o mercantiles, lugar donde la literatura económica ha sido más vasta (Julien 1998, Szarka 1998). Las relaciones se establecen a partir de la red que se genera en los procesos de transacciones comerciales, ya que comprenden intercambios monetarios, de mercancías, financieros. "El núcleo de la red de intercambio está constituido por las contrapartes de negocios de la empresa, es la red de producción” (Julien 1998:26). Esta red está conformada por los proveedores, los clientes, instituciones financiadoras tanto de crédito formal o informal.

En resumen, las redes se ponen en movimiento teniendo en cuenta ciertos aspectos estructurales, que son punto de partida y punto de llegada en la forma en que se generan, destruyen o consolidan determinado tipo de relaciones, de acuerdo a los miembros insertos en las redes. Estos aspectos estructurales lo constituyen tanto los recursos económicos y la envergadura del capital puesto en la unidad económica, como los recursos no tangibles que permiten el desarrollo de un tipo de actividad y no otro, y que están constituidos por los acerbos, las capacidades, las habilidades y las credenciales educativas formales, constitutivos del capital social.

\section{Aportes y aprestos teóricos sobre la confianza}

Varios autores han planteado (Granovetter 1985, Portes 1998, Putnam 2002, Coleman, 1990, Laacher 2004, Derbel y Mamlouk 2003, Luhman 2005), desde diversas perspectivas el rol de la confianza como un componente importante en el proceso de garantizar interacciones a través del tiempo, y rol preponderante en la constitución de capital social, y del fortalecimiento de las redes sociales.

Sin embargo, pocas veces en las ciencias sociales latinoamericana se le ha dado el estatus teórico de relevancia a la confianza para explicar o entender determinado tipo de prácticas sociales.

Es en ese sentido que el presente artículo quiere rescatar y buscar los componentes centrales que asoman en un proceso que partió con el supuesto de que la confianza constituye un elemento central cuando otras formas que actuarían como garante de las interacciones aparecen débiles, ausentes o no primordiales en un contexto de garantía de las interacciones sociales, como es el caso de la feria de Solano. Por otro lado el eje está 
puesto en los procesos de cooperación, continuidad y perdurabilidad de las interacciones, y no tanto desde el conflicto, la lucha, la confrontación, aunque están presentes y constituyen los espacios de confrontación y asignación de roles en el campo de la feria.

\section{Algunos antecedentes sobre la confianza}

La problemática de la cooperación ha sido tratada en el marco del análisis de los dilemas sociales (en particular el dilema del prisionero y la teoría de los juegos), y en menor medida cuando se ha analizado el tema de la confianza.

La idea es que en una organización cualquiera, los agentes no cooperan simplemente en hechos de confianza. Hay otros elementos que entran en juego cuando una persona se compromete en una relación de interdependencia con otra, sobre todo cuando esta última experimenta un débil acuerdo con su interlocutor. No se puede definir la confianza sin expresar que sienten realmente los sujetos por ese vocablo. La confianza no se inscribe en el dominio de la ficción y de la ilusión, es un fenómeno que se inscribe en el universo de la realidad y del enigma.

Karipik, citado por Walid Derbel y Zeineb Ben Ammar Mamlouk dice: “Colocar su confianza en alguien o alguna cosa, dejarse guiar por una realidad exterior al menos particularmente impenetrable, abandonarse en alguna suerte de autoridad extraña, no poder encontrar su justificación en un solo saber verificable: ninguno conoce previa y directamente en forma suficiente la garantía de su acción presente o futura”. (Walid Derbel y Zeineb Ben Ammar Mamlouk 2003: 4)

La confianza y la cooperación, como se postula en este trabajo, son aquí los términos que serán utilizados continuamente para representar la síntesis de una sensación de emoción y de conocimientos que cada individuo se apropia, aprehende y genera a su modo, el problema de la cooperación es ante todo, un problema de confianza. (Derbel y Mamlouk, 2004)

\section{La cuestión de la confianza}

En base a las principales prácticas observadas en la feria, se aprecian algunos ejes observables de la confianza:

- La confianza como un atributo personal (entendida como un caso específico ligado a una o varias personas, que actúan basadas particularmente en las actitudes personales independientemente del conjunto de otras aptitudes.)

- La confianza como un comportamiento (se comprende como el fenómeno extendido por medio de un grupo de personas o un colectivo. El 
tipo de comportamiento reproducido es similar en todos los miembros de un mismo grupo.)

- La confianza como una figura situacional. (El fenómeno de confianza es imperativamente un punto de pasaje independiente de las personas que están presentes en este género de situación)

- La confianza como un arreglo institucional. (Una lógica contractual entre los actores, una negociación de comportamiento, sobre un acuerdo o un compromiso en presencia de los están en el juego). nalidad.

a) La confianza como un atributo personal o un tratado de la perso-

Esta primera tipología está referida a una concepción de la confianza dominada por un aspecto psicológico: La confianza es definida en términos de expectativas confidenciales positivas para con la conducta de otra persona, mientras que la desconfianza refleja la expectativa confidencial negativa (Lewiki 1998). Bajo esa idea la confianza aparece como un atributo personal, en el sentido que ella se interroga sobre la existencia de una calidad apropiada para el individuo, y que no puede ser portada colectivamente. El alcance de la confianza de una persona es su voluntad de acción sobre la base de las palabras, acciones y decisiones de otro (McAllister, 1995).

La confianza también es vista como una emoción. Ciertos autores hablan de la confianza como una premonición de seguridad sin reserva. Seguridad confidencial sobre la integridad, la honestidad o la justicia de otro, como la garantía sobre la credibilidad de una persona u otro objeto con renunciamiento a toda forma de prudencia y de investigación. (Derbel y Mamlouk, 2003)

También la confianza es vista como una intención o creencia. Otras definiciones acentúan sobre dos aspectos más conocidos del concepto, en términos de un atributo personal: primero ponen el acento sobre las creencias y segundo sobre las intenciones. Por ejemplo, Mc Knightt concibe la confianza simplemente como "la creencia de alguien y su voluntad de depender de otra persona” (McKnight, 1998 en Derbel y Mamlouk, 2003).

Derbel y Mamlouk retoman estos dos ejes centrales: la confianza expresada como una creencia que el otro tiene de las intenciones positivas de nuestra acción. Es la buena voluntad y el cuidado para con nuestra consideración en la acción. La creencia que el otro es componente, es apto a procurar su atención, una suerte de anticipación, la otra persona es madura, capaz de hacer pruebas, de jugar un rol de responsabilidad. (Derbel y Mamlouk, 2003). Sin embargo, explicar la confianza en términos de expectativa, reduce el campo de análisis a una percepción individual y eclipsa en particular el valor comportamental de su representación. 
Este tipo de confianza, sitúa la interacción en la feria a partir de ciertos rasgos que convalidan y legitiman el intercambio: la amistad entre los que realizan la transacción, la generación de imágenes que garantizan esa relación: la forma de vestir, el buen trato al relacionarse, la mirada, el apretón de manos, el amigo de un amigo, el origen territorial, la forma de hablar, el compartir gustos por equipos de fútbol, entre otras situaciones.

b) La confianza es la expresión de un comportamiento honesto.

La confianza puede ser definida, además, sobre la base de que se puede demostrar que una persona es coherente y previsible en sus acciones en el curso de su vida social. La confianza es por lo tanto un testimonio comportamental, es una apreciación proveniente del exterior, y una certificación pública. (Derbel y Mamlouk, 2003), bajo esta expresión se entienden dos formas de confianza ligada al comportamiento:

La confianza experimentada en términos de líneas de conducta, de acciones y de decisiones. En este cuadro, la confianza es instituida sobre la base de un aforismo cognitivo basado sobre los antecedentes de una persona y su capacidad futura a considerar las normas de conductas sociales. "La confianza es la expectativa que una persona o una entidad manifiesta un comportamiento justificado sobre un plan ético. Este comportamiento implica toda decisión y acción resultante de la adhesión a un conjunto de principios (reglas de conducta).(Hosmer, 1995 en Derbel y Mamlouk, 2003)

La confianza experimentada en términos de palabra dada. La confianza es asociada a la palabra y a la credibilidad del compromiso. Ella se revela como coherente entre el decir y el hacer. La confianza debe ser sentida como un juego personal y confidencial sobre las intenciones y móviles de otra persona y sobre la sinceridad de sus palabras.

Este es el lugar de la experiencia, que "certifica” en base a las prácticas, la confianza social. Es el lugar del "compadre”, el buen vecino, el protector político, siempre desde un ámbito de proximidad; es el cliente regular, los miembros del "pasanako"”, que se presentan como los componentes que sellan pactos a través del fiado, el crédito, el feriante que ofrece regular y cotidianamente sus productos, y garantiza la calidad de ellos, el comprador que elige ese puesto regularmente y compra allí. Todos esos

\footnotetext{
${ }^{1}$ La fuente propia de financiamiento de los feriantes, es el "pasanako” o cadena crediticia, que consiste en la agrupación de varios feriantes que colocan en un «pozo» común semanal una determinada cantidad de dinero (cantidad de semanas de acuerdo a los integrantes del círculo de ahorro), y sortean el orden en los cuales los integrantes uno por vez, se llevan ese «pozo». Existe una variante, en la que se le agrega intereses a este pozo, para resolver el tema de la inflación. La garantía de cumplimiento está dada por las relaciones cara a cara de los feriantes, el compadrazgo y la amistad o el compañerismo de largos años de conocerse en la feria. El no cumplimiento implica el desprecio y el aislamiento social de los otros feriantes que pesa coercitivamente sobre quien escapa a estas reglas no escritas.
} 
vínculos generan prácticas de confianza basados principalmente en la experiencia , en el acontecer de sus continuas intervenciones.

c) La confianza como la expresión de una situación de precariedad.

Las características fundamentales de esta descripción, es que la confianza interpela los factores que hacen presión sobre la situación, más que interpelar los actores que están implicados. La confianza como la persistencia de la situación, donde la confianza es el conjunto de las expectativas compartidas por el conjunto de los participantes en un intercambio, y ellos se mantienen hasta que son violados. (Derbel y Mamlouk, 2004). Es como que se 'puede considerar a la confianza como expresión de las situaciones de riesgo, de daños y de oportunismo; para que la confianza sea realmente pertinente, debe tener la posibilidad de terminar por una decepción o una traición, (Gambetta D. en Derbel y Mamlouk, 2004. Desde esta perspectiva la confianza implica situaciones donde al menos una de las partes experimenta una ganancia significativa y es consciente del potencial de traición o de daño que puede causar a la otra parte.

Derbel y Mamlouk plantean que la confianza es una aceptación del riesgo asociado al tipo y a la profundidad de la interdependencia en una relación dada. Por ejemplo el feriante de conservas sin autorización sanitaria, del cual se desconoce la forma de la producción, vende su producto a un cliente, quien tiene interés en aceptar una oferta del producto para satisfacer su apetito y su gusto por la conserva. Pero, ¿puede aceptar cualquier producto, cualquier conserva?, él tiene confianza y es una alternativa ante la posibilidad de satisfacer su deseo de comerla.

También puede estimarse que la confianza juega un rol esencialmente de orden preventivo, ello permite a las personas protegerse contra el riesgo potencial de una desviación comportamental de otro. Derbel y Mamlouk sostienen que la confianza es una seguridad mutua, que algunas de las partes en un intercambio no tendería a explotar la vulnerabilidad del otro (Derbel y Mamlouk, 2003), por lo cual en ausencia de vulnerabilidad, el concepto de la confianza no es de suficiente peso significativo sobre el intercambio.

Esta situación de precariedad en la feria, que se da a través de la venta de alimentos elaborados, por ejemplo: empanadas, pan con chorizos, tortillas al carbón, entre otros platos, que no cumplen ningún requisito formal establecido por las autoridades sanitarias para la elaboración y venta de alimentos, sin embargo sólo la confianza en el vendedor, y sus productos, estimula la transacción, suplanta la regulación, o mejor dicho la reemplaza cuando esta no existe.

d) La confianza es un arreglo institucional.

La confianza traduce una forma de negociación, implícita o explícita. Ella juega un rol regulador. Ella permite a los participantes producir por 
su cuenta espacios de libertad de acción y velar por el respeto por el otro. La confianza en términos de arreglos institucional experimenta un proceso de socialización fundada sobre el juego de la interdependencia. La acción de uno pone en consideración las posibilidades de reacción del otro. (Derbel y Mamlouk, 2003)

Se puede distinguir dos formas de confianza institucional:

- La confianza en términos de predicción, como predicción racional, en la que los agentes ponen el acento sobre la obtención y análisis de información a fin de proyectar los eventuales consecuencias futuras de ciertos eventos.

- La confianza en términos de especulación. Donde la confianza puede traducirse en un sentido del cálculo, una suerte de optimización relacional en la medida donde un individuo no elige sus colaboradores de modo arbitrario; al contrario esta elección está fundada sobre el cálculo que reduce la incertidumbre y el daño.

Es el proceso formal de los acuerdos, es “el arreglo institucional de la confianza”, son los pactos estables y regularizados con la práctica habitual y cotidiana: el permiso informal de la ocupación de espacios en la feria, el lugar de la mutual, que implica cumplir mis compromisos y asegurar mi reproducción.

Todos estos aspectos no son formas lineales ni jerárquicas de la confianza, sino modos en que la misma aparece, reemplazando o conviviendo con otras formas de confianza.

\section{La cuestión de la cooperación}

Para aprehender la cooperación, y entendiéndola como un aporte conceptual a la dinámica de la feria, conviene situarla dentro de un contexto de la interacción social.

“...Este orden social no es otra cosa que la expresión de una configuración social creada bajo la empresa de las interacciones naturales y frecuentes entre los miembros de una misma colectividad. Un orden se crea, de las leyes de los principios y de los valores que resumen la acción de todos en acción organizada o acción colectiva.” (Derbel y Mamlouk, 2003: 41). Es el seno de esta acción colectiva que la cooperación actúa.

Siguiendo esta línea argumental la cooperación es una acción deseada y provechosa, benéfica y buscada, y en ese sentido la cooperación depende fuertemente de un campo social de interacción, imperativamente puntual, temporal y acotado. La cooperación es una expresión de un dilema, el concepto de cooperación tiene por tanto a ser introducido en el marco del análisis de los dilemas sociales, y en particular el ha sido integrado al célebre dilema del prisionero, donde dos de los prisioneros deben jugar, son llamados a hacer una elección racional, entre cooperar con su compañero o denunciar a su compañero y co- 
operar con la justicia. El dilema del prisionero es bastante notorio al describir una situación que enfrenta y orienta las posibilidades de acción en función de dos alternativas, la cooperación o la denuncia.

Según algunos autores (Lewicki 1998, Derbel y Mamlouk, 2003) la cooperación de los actores y autores de los problemas y de sus soluciones es por lo tanto siempre sostenida por los reportes de dependencia y de poder, es decir de las relaciones de intercambio negociada, generalmente heterogéneas .Toda vez y en todo momento ella puede ser comprendida como un conjunto de juegos donde las reglas y las convenciones (formales e informales- explicitas o tácitas) disciplinan las tendencias oportunistas de los actores y canalizan, regularizan y se hacen más previsibles.

El análisis de la confianza como base de la cooperación nos da un marco de comprensión, por lo menos conceptual, de la garantía de los intercambios, la perdurabilidad de los mismos, y la dimensión que asumen, en un contexto particular: la feria. Pero es necesario aclarar ciertas ideas.

Primeramente, los actores no son totalmente independientes. Las interacciones improvisadas dan nacimiento a una estructura de interdependencia social compleja que escapa a los organizadores. En efecto, los actores descubren la existencia de un campo libre de acción, y en función de su percepción de la envergadura de esta libertad, buscan registrar una parte de sus acciones en comportamientos de intereses específicos. Pero rápidamente, estos actores se dan cuenta que, sin la complicidad de otros actores, las ganancias son magras. Esta complicidad, no puede ser arbitraria, ella cuanta sobre la capacidad de cada actor a hacer confianza con el otro. (Derbel y Mamlouk, 2003)

Esto es precisamente lo que la lógica de juego, y de la cooperación deviene determinante. Los actores son libres de actuar en concordancia con la lógica del sistema formal y, en las apariencias, esta libertad no se opone a las reglas organizacionales de la feria, pero paralelamente, estos mismos actores, y sobre la base de un sistema de cooperación más o menos elaborado, juegan con estas reglas y las utilizan en función de sus intereses, principios o caprichos.

Toda la cuestión vuelve entonces a interrogarse sobre la capacidad de los actores a jugar con las reglas formales, a explotar lo impreciso para colocar en su lugar una estrategia individual (sin cooperación) o colectiva (con cooperación). La segunda idea retiene que los sistemas organizacionales, como la feria, compuestos por una parte estructurada, y otra vaga, marginal, informal, permite afrontar los conflictos de intereses y encontrar equilibrios a través de la cooperación o la colaboración de los actores.

\section{A modo de cierre}

Es en ese sentido que las páginas precedentes han sido un intento de exponer un proceso de articulación entre valores y prácticas sociales, ubi- 
cadas en un contexto de producción, distribución y consumo en un mercado callejero: la feria.

Allí confluyen múltiples racionalidades culturales, principalmente, expresadas en sus trayectorias y en sus miradas del "mundo", en las que la economía de esas prácticas se posiciona de forma diversa, se ajusta, se encastra, como plantea Polanyi, en instituciones de diversa índole, con diferente grado de formalidad explícito o no, para lograr las mejores condiciones para la supervivencia.

En ese sentido se recupera el concepto de las relaciones sociales, las cuáles están impregnadas de valores, de poder, de cosmovisiones y no pueden ser meramente analizadas como relaciones de individuo a individuo, como lo plantea la filosofía liberal. Y como argumenta Marx en la Miseria de la filosofía (Marx, [1847]), "las relaciones sociales de producción, producen la sociedad en todos sus órdenes, a partir de múltiples y recíprocas relaciones entre infraestructura y superestructura”. Por lo cual se trata de rescatar "la solidaridad entre la teoría y la praxis, entre los sistemas de ideas y las formas de apropiación material de la naturaleza y las relaciones sociales que se crean y reproducen estos procesos” (Marx, 1999).

El individuo calculador, objeto del pensamiento económico y político moderno, es relativo e histórico, incluyendo sus intereses, ya que es un producto social.

Bourdieu dice en su libro Las estructuras sociales de la economía que "la ciencia que llamamos economía se sustenta en una abstracción inicial que consiste en disociar una categoría particular de prácticas, o una dimensión particular de cualquier práctica, del orden social en que toda práctica está inmersa (Bourdieu, 2005: 55).

Pero no basta establecer las relaciones entre valores, cosmovisiones, trayectorias, y prácticas económicas, sino, como dice Polanyi, hacen falta “estructuras de apoyo”, complejos institucionales que trascienden las acciones de los sujetos. Es lo que Polanyi llama formas de integración, que se entienden como los marcos contextuales en los que se realizan los intercambios de bienes, servicios y personas, desde una perspectiva "sustantiva".

Y es allí donde juega y asume un papel primordial, lo que Granovetter denomina “inserción”, en la que uno de los pocos recursos contra las transgresiones, o la incertidumbre de los intercambios, es la confianza mutua que resulta de la pertenencia a una estructura social que los abarca, y que en este caso es la feria. Como se pudo observar, en el intercambio de carácter informal, la confianza es generada tanto por las características y sentimientos comunes de las personas y por las expectativas de las mismas, si no son cumplidas serán castigadas mediante la exclusión de quien haya violado los pactos, de las redes en las cuales estaban insertos.

Es ahí donde comenzamos a dar respuesta al problema principal de 
este artículo, de cómo pueden preservarse, expandirse y prolongarse las estrategias de sobrevivencia de los feriantes de San Francisco Solano, sobre todo ante la ausencia o presencia híbrida de mecanismos regulatorios públicos.

Y es por ello que la confianza, bajo la idea de construcción continua de los procesos interactivos sociales en un contexto especial y espacial -la feria-, asume un rol central. Las actividades informales, llevadas a cabo por feriantes, profundamente segmentados al interior del mercado callejero de Solano, parecerían regladas por estos acuerdos tácitos que alimentan procesos de cooperación entre los feriantes, y regulan los conflictos latentes, de la "lucha" por el territorio: el espacio de la feria, y el cliente potencial. 


\section{Bibliografía}

Alexander, J. (1997), Las teorías sociológicas desde la Segunda Guerra Mundial. Análisis miltidimensional. Edi. Gedisa, Barcelona, España.

Bourdieu, P. (1988), La distinción. Criterio y bases sociales del gusto. Editorial Taurus, Buenos Aires, Argentina.

Bourdieu, P. Chamboredon J. C., Passeron J.C. (2004), El oficio del Sociólogo, presupuestos epistemológicos, Siglo Veintiuno, editores Argentina, Buenos Aires.

Bourdieu, P. (2005), Las estructuras sociales de la economía, Editorial Manatial, Buenos Aires, Argentina.

CEPAL (1997), "La brecha de la equidad. América Latina, y el Caribe”. Primera Conferencia Regional de Seguimiento de la Cumbre Mundial sobre Desarrollo Social, 6 al 9 de abril de 1997. LC/G.1954 (Conf.86/3).Sao Paulo, Brasil.

Coleman, J. (1990), Foundations of Social Theory.: The Belknap Press of Harvard University Press. Cambridge, Masachussetts.

Derbel W., y Ben Ammar Mamlouck Z. (2003), “Le dilemme de la confiance et de la coopération: interdepéndance des acteurs et supremátie du systéme organisationel” en La Revue, Nov/Dec 2003, París, Francia.

Espinoza, Vicente (1998), “El capital social: Oportunidades para superar la pobreza, Potencialidades y oportunidades: una perspectiva comprensiva de la pobreza y su medición”. Encuesta sobre capital social y redes sociales de las familias chilenas, Capítulo V, documento de trabajo, Departamento de Economía, Universidad de Chile y Consejo Nacional para la Superación de la Pobreza, Santiago de Chile.

Giddens, A. (1986), La constitución de la sociedad: bases para la teoría de la estructuración. Buenos Aires: Amorrutu.

Granovetter, M. (1985), “Economic Action and Social Structure: The Problem of Embeddedness”, American Journal of Sociology, 91, (pp481510), New Jersey.

Julien P. A. (1998), “Las Pequeñas empresas como objeto de investigación: algunas reflexiones acerca del conocimiento de las pequeñas empresas y sus efectos sobre la teoría económica”, en Kantis Hugo (ed.) Desarrollo y Gestión en PyMES, Universidad Nacional de General Sarmiento, (pp. 27-46) Buenos Aires.

Kaztman, Rubén; Beccaria, Luis; Filgueira, Fernando; Golbert, Laura; Kessler, Gabriel (1999), Vulnerabilidad, activos y exclusión social en Ar- 
gentina y Uruguay. Santiago de Chile: OIT; Fundación Ford.

Laacher, S. (2004), “Los sistemas de intercambio local”. Revista lavboratorio $N^{\circ} 16$, Buenos Aires, Argentina

Lewiki, R. (1998), Trust and disfrut: new relationships and realities (especial topic forum on trust in and betwen organizations), Academy of management Review Kentucky, USA.

Lin, N. (2001), Social Capital: A theoty of social estructure and action. Cambrigde University Press, Nueva York.

Luhmann N. (2005), Confianza , Editorial Antrophos, Barcelona, España.

Marx, C. (1994), El Capital, Tomo 1, Volumen 1, Siglo XXI Editores, México.

Mcallister D. J. (1995), Affect and cognitiosn based trust as foundation for interpersonnal cooperation in organization, Acadamey of managament Review, Kentucky, USA.

Portes, A. (1998), Social Capital: Its Origins and Applications in Modern Sociology. En Annual Review of Sociology, New Jersey.

Putnam, R, Robert L. y Raffaella Y. Nanetti. (1994). Para que la democracia funcione. Tradiciones cívicas en Italia. Edit. Galas, Caracas.

Putnam, R. (2002), Solo en la bolera. Galaxia Gutenberg, Círculo de Lectores. Barcelona, España.

Szarka, J. (1998), “Las Redes y la Pequeña Empresa”, en Kantis Hugo (ed.) Desarrollo y Gestión en PyMES, Universidad Nacional de General Sarmiento (pp. 309-328) Buenos Aires.

Val Burris (1993), La síntesis neomarxista de Marx y Weber sobre las clases. Mimeo, Facultad de Ciencias Sociales, Universidad de Buenos Aires.

Weber, M (1993), Economía y sociedad: Esbozo de una sociología comprensiva. Fondo de Cultura Económica, México. 